\title{
NREL Photovoltaic Program FY 1993 Bibliography
}

\author{
L. Pohle, Editor
}

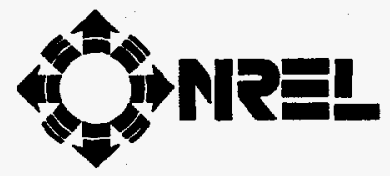

National Renewable Energy Laboratory 1617 Cole Boulevard

Golden, Colorado 80401-3393

A national laboratory of the U.S. Department of Energy Operated by Midwest Research Institute for the U.S. Department of Energy under contract No. DE-AC02-83CH10093

Prepared under Task No. PV410101

January 1994 


\section{NOTICE}

NOTICE: This report was prepared as an account of work sponsored by an agency of the United States government. Neither the United States government nor any agency thereof, nor any of their employees, makes any warranty, express or implied, or assumes any legal liability or responsibility for the accuracy, completeness, or usefulness of any information, apparatus, product, or process disclosed, or represents that its use would not infringe privately owned rights. Reference herein to any specific commercial product, process, or service by trade name, trademark, manufacturer, or othenwise does not necessarily constitute or imply its endorsement, recommendation, or favoring by the United States government or any agency thereof. The views and opinions of authors expressed herein do not necessarily state or reflect those of the United States government or any agency thereof.

Printed in the United States of America

Available from:

National Technical information Service

U.S. Department of Commerce

5285 Port Royal Road

Springfield, VA 22161

Price: Microfiche $A 01$

Printed Copy $\mathrm{A03}$

Codes are used for pricing all publications. The code is determined by the number of pages in the publication. Information pertaining to the pricing codes can be lound in the current issue of the following publications which are generally available in most libraries: Energy Research Abstracts (ERA); Government Reports Announcements and index (GRA and I): Scientific and Technical Abstract Reports (STAR); and publication NTIS-PR-360 available from NTIS at the above address. 


\section{DISCLAIMER}

Portions of this document may be illegible electronic image products. Images are produced from the best available original document. 


\section{NREL PHOTOVOLTAIC PROGRAM \\ Bibliography \\ October 1992 - September 1993}

Alonso, R. G.; Mascarenhas, A.; Froyen, S.; Horner, G. S.; Bertness, K.; Olson, J. M. (January 1993). "Polarized Piezomodulated Reflectance Study of Spontaneous Ordering in GaInP2." Solid State Communications (85:12); Pergamon Press Ltd, Great Britain; pp 1021-1024. NREL/TP-451-5166.

Arent, D. J.; Bode, M.; Bertness, K. A.; Kurtz, S. R.; Olson, J. M. (April 1993). "Band-Gap Narrowing in Ordered Ga0.47In0.53As." Applied Physics Letters (62:15); American Institute of Physics; pp. 1806-1808. TP-451-5128.

Armstrong, J. M.; Misra, M. S.; Lanning, B. (March 1993). Innovative Sputtering Techniques for CIS and CdTe Submodule Fabrication, Annual Subcontract Report, 1 September 1991 31 August 1992. SERI/TP-413-5406. 62 pp. Work performed by Martin Marietta, Denver, Colorado. Available NTIS: Order No. DE93000085.

Arya, R.; Fogleboch, J.; Lommasson, T.; Podlesny, R.; Russell, L.; Skibo, S.; Wiedeman, S.; Rothwarf, A.; Birkmire, R. (September 1993). Research on Polycrystalline Thin-Film Submodules Based on CuInSe2 Materials, Annual Subcontract Report, 1 November 1991 31 December 1992. SERI/TP-413-5759. 74 pp. Work performed by Solarex Thin Film Division, Newtown, Pennsylvania. Available NTIS: Order No. DE93018223.

Baron, B. N.; Birkmire, R. W.; Phillips, J. E.; Shafarman, W. N.; Hegedus, S. S.; McCandless, B. F. (October 1992). Polycrystalline Thin Film Materials and Devices, Annual Subcontract Report, 16 January 1991 - 15 January 1992. SERI/TP-451-5094. $106 \mathrm{pp}$. Work performed by Institute of Energy Conversion, Newark, Delaware. Available NTIS: Order No. DE93000022.

Basol, B. M.; Kapur, V. K.; Halani, A.; Leidholm, C. (October 1992). Low-Cost CulnSe2 Submodule Development, Final Subcontract Report, 9 July 1990 - 31 January 1992. SERI/TP-413-5010. 38 pp. Work performed by International Solar Electric Technology, Inglewood, California. Available NTIS: Order No. DE92016438.

Bennett, M. S.; Newton, J.; Poplawski, C.; McMahon, T. J. (1993). "An Investigation of the Effect and Nature of Shunts in a-Si Solar Cells." Conference Record of the 23rd IEEE PV Specialists Conference, May 10-14, 1993, Louisville, KY. 
Benson, D. K.; Burrows, R. W., Inventors (April 1993). Microwave Impregnation of Porous Materials with Thermal Energy Storage Materials, U.S. Patent No. 5,202,150. 16 pp. Assignee: U.S. Department of Energy.

Benson, D. K.; Potter, T. F., Inventors (October 1992). Compact Vacuum Insulation, U.S. Patent No. 5,157,893. 24 pp. Assignee: Midwest Research Institute, Kansas City, Missouri.

Birkmire, R. W.; Phillips, J. E.; Shafarman, W. N.; Hegedus, S. S.; McCandless, B. E.; Yokimcus, T. A. (August 1993). Polycrystalline Thin Film Materials and Devices, Final Subcontract Report, 16 January 1990 - 15 January 1993. SERI/TP-451-5755. 135 pp. Work performed by the Institute of Energy Conversion, University of Delaware, Newark, Delaware. Available NTIS: Order No. DE93018213.

Birkmire, R. W.; McCandless, B. E.; Yokimcus, T. A.; Mondal, A. (October 1992). Novel Ways of Depositing ZnTe Films by a Solution Growth Technique, Final Subcontract Report, 1 January 1990 - 1 January 1992. SERI/TP-410-4959. 78 pp. Work performed by Institute of Energy Conversion, University of Delaware, Newark, Delaware. Available NTIS: Order No. DE92010591.

Bottenberg, W. R.; Hall, R. B.; Jackson, E. L.; Lampo, S.; Mulligan, W. E.; Barnett, A. M. (April 1993). Silicon Film(tm) Photovoltaic Manufacturing Technology, Semiannual Technical Progress Report, 15 January 1992 - 15 July 1992. SERI/TP-411-5455. 27 pp. Work performed by AstroPower, Inc., Newark, Delaware. Available NTIS: Order No. DE930100008.

Branz, H. M.; Schiff, E. A. (1993). "Dangling-Bond Relaxation and Deep-Level Measurements in Hydrogenated Amorphous Silicon." Physical Review B, Vol. 48, pp. 8667-8671.

Branz, H. M.; Asher, S. E.; Nelson, B. P. (March 1993). "Light-Enhanced Deep Deuterium Emission and the Diffusion Mechanism in Amorphous Silicon." Physical Review B (47:12); Ameriacan Physical Society; pp. 7061-7066. NREL/TP-451-4726.

Cannon, T. (November 1992). "Recent Advances in Solar Radiometry at the National Renewable Energy Laboratory." SPIE Volume 1712, Proceedings of the 14th Symposium on Photovoltaic Measurements, Sopron, Hungary, 1992.

Cannon, T.; Hulstrom, R.; Trudell, D. (May 1993). "New Instrumentation for Measuring Spectral Effects During Indoor and Outdoor PV Device Testing." Conference Record of the 23rd IEEE PV Specialists Conference. 
Ciszek, T. F.; Burrows, R. W.; Wang, T. H. (October 1992). "High Temperature Solution Growth of Thin Crystalline Silicon Layers." Eleventh E.C. Photovoltaic Solar Energy Conference Proceedings, Proceedings of the International Conference, 12-16 October 1992, Montreux, Switzerland. Harwood Academic Publishers, Montreux, Switzerland; pp. 423-426. SERI/TP-451-4783.

Cohen, J. D. (August 1993). Microscopic Origins of Metastable Effects in a-Si:H and Deep Defect Characterization in a-Si,Ge:H Alloys, Annual Subcontract Report, 1 February 1992 - 31 January 1993. SERI/TP-451-5737. 29 pp. Work performed by University of Oregon, Eugene, Oregon. Available NTIS: Order No. DE93018205.

Collins, R. W.; Wronski, C. R.; An, J.; Li, Y. (December 1992). In-Situ Characterization of Growth and Interfaces in a-Si:H Devices, Annual Subcontract Report, 1 May 1991 - 30 April 1992. SERI/TP-451-5265. 59 pp. Work performed by Pennsylvania State University, University Park, Pennsylvania. Available NTIS: Order No. DE93000051.

Contreras, M.; Tuttle, J.; Noufi, R. (1993). "Validation of an In-Line System for Large-Scale

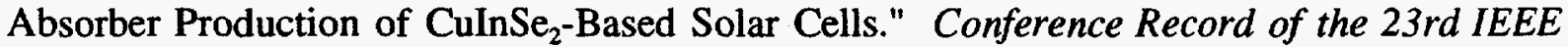
PV Specialists Conference, May 10-14, 1993, Louisville, KY.

Contreras, M.; Tuttle, J.; Du, D.; Qu, Y.; Swartzlander, A.; Tennant, A.; Noufi, R. (September 1993). "Graded Band-Gap $\mathrm{Cu}(\mathrm{In}, \mathrm{Ga}) \mathrm{Se}_{2}$ Thin-Film Solar Cell Absorber with Enhanced Open-Circuit Voltage." Applied Physics Letters, 63(13), pp. 1824-1826.

Coutts, T. J.; Lundstrom, M. S. (January 1993). "Research Opportunities in Crystalline III-V Photovoltaics." SERI/TP-413-5062. Journal of Electronic Materials (22:1); pp. 57-64. TP-413-5062.

Dagan, G.; Ciszek, T. F.; Cahen, D. (December 1992). "Ion Migration in Chalcopyrite Semiconductors." Journal of Physical Chemistry (96); American Chemical Society; pp. 11009-11017.

Dapkus, P. D. (February 1993). Low Temperature Metal-Organic Chemical Vapor Deposition Growth Processes for High Efficiency Solar Cells, Final Technical Report, 1 September 1985 - 30 November 1989. SERI/TP-451-4853. $235 \mathrm{pp}$. Work performed by the University of Southern California, Los Angeles, California. Available NTIS: Order No. DE92010562.

Dapkus, P. D.; Hummel, S. G. (August 1993). High Efficiency Epitaxial Optical Reflector Solar Cells, Final Subcontract Report, 1 January 1990 - 31 October 1992. SERI/TP-451-5591. 51 pp. Work performed by the University of Southern California, Los Angeles, California. Available NTIS: Order No. DE93017115. 
Delahoy, A. E.; Britt, J.; Kiss, Z. (February 1993). Non-H2Se, Ultra-Thin CIS Devices, Annual Subcontract Report, 10 March 1992 - 9 November 1992. SERI/TP-413-5334. 69 pp. Work performed by Energy Photovoltaics, Inc., Princeton, New Jersey. Available NTIS: Order No. DE93000078.

Duran, G.; Mackamul, K.; Metcalf, D.; Stern, M.; Volltrauer, H.; Varar, T.; Urbanski, E.; DiBiaso, T.; Skinner, D. (September 1993). Amorphous Silicon PVMaT Phase 2A, Semiannual Subcontract Report, 1 May 1992 - 31 October 1992. SERI/TP-411-5739. 49 pp. Work performed by Utility Power Group, Chatsworth, California, and Advanced Photovoltaic Systems, Inc., Princeton, New Jersey. Available NTIS: Order No. DE93018221.

Fortmann, C. M.; Hegedus, S. S. (December 1992). Stable, High-Efficiency Amorphous Silicon Solar Cells with Low Hydrogen Content, Annual Subcontract Report, 1 March 1991 - 31 January 1992. SERI/TP-451-5191. $70 \mathrm{pp}$. Work performed by the Institute of Energy Conversion, Newark, Delaware. Available NTIS: Order No. DE93000049.

Fragalli, J. F.; Misoguti, L.; Nakagaito, A. N.; Grivickas, V.; Bagnato, V. S.; Branz, H. M. (1993). "Hydrogenated Amorphous Silicon by 60-Hz Glow-Discharge Deposition." Journal of Applied Physics, Vol. 74, pp. 668-671.

FY 1992 Measurements and Characterization Branch Annual Report. (March 1993). SERI/TP-412-5422. 30 pp. Available NTIS: Order No. DE93000094.

FY 1991 Measurements and Characterization Branch Annual Report. (November 1992). SERI/TP-412-5160. 54 pp. Available NTIS: Order No. DE93000030.

Gallagher, A.; Ostrom, R.; Stutzin, G.; Tanenbaum, D. (February 1993). Growth Mechanisms and Characterization of Hydrogenated Amorphous-Silicon-Alloy Films, Annual Subcontract Report, 14 February 1991 - 13 February 1992. SERI/TP-411-5326. 18 pp. Work performed by National Institute of Standards and Technology, Washington, DC. Available NTIS: Order No. DE93000071.

Gallagher, A.; Tanenbaum, D.; Laracuente, A.; Molenbroek, E. (August 1993). Growth Mechanisims and Characterization of Hydrogenated Amorphous-Silicon-Alloy Films, Annual Subcontract Report, 14 February 1992 - 13 February 1993. SERI/TP-411-5749. 29 pp. Work performed by National Institute of Standards and Technology, Boulder, Colorado. Available NTIS: Order No. DE93018202.

Gallium Arsenide-Based Ternary Compounds and Multi-Band-Gap Solar Cell Research, Annual Subcontract Report, 15 April 1988 - 14 June 1990. (February 1993). SERI/TP-451-5333. $170 \mathrm{pp}$. Work performed by Spire Corporation, Bedford, Massachusetts. Available NTIS: Order No. DE93000084. 
Ghosh, M.; Kampas, F.; Xi, J. (September 1993). Research on Stable, High-Efficiency Amorphous Silicon Multijunction Modules, Annual Subcontract Report, 1 November 1992 - 31 May 1993. SERI/TP-411-5760. 18 pp. Work performed by Advanced Photovoltaic Systems, Princeton, New Jersey. Available NTIS: Order No. DE93018220.

Ghosh, M.; DelCueto, J.; Kampas, F.; Xi, J. (February 1993). Research on Stable, High-Efficiency Amorphous Silicon Multijunction Modules, Annual Subcontract Report, 1 December 1991 - 31 October 1992. SERI/TP-411-5327. 13 pp. Work performed by Advanced Photovoltaic Systems, Princeton, New Jersey. Available NTIS: Order No. DE93000072.

Gordon, R. G. (April 1993). Optimization of Transparent and Reflecting Electrodes for Amorphous Silicon Solar Cells, Annual Subcontract Report, 1 May 1991 - 30 April 1992. SERI/TP-411-5456. $105 \mathrm{pp}$. Work performed by Harvard University, Cambridge, Massachusetts. Available NTIS: Order No. DE93010009.

Gratzel, M.; Munavalli, S.; Pern, F.-J.; Frank, A. J., Inventors (June 1993). Molecular Water Oxidation Catalyst, U.S. Patent No. 5,223,634. 18 pp. Assignee: U.S. Department of Energy.

Green, M. A.; Emery, K. (January 1993). "Solar Cell Efficiency Tables." Progress in Photovoltaics: Research and Applications (1:1); pp. 25-29. NREL/TP-412-5122.

Guha, S. (July 1993). Research on Stable, High-Efficiency Amorphous Silicon Multijunction Modules, Annual Subcontract Report, 1 January 1992 - 28 February 1993. SERI/TP-411-5654. 72 pp. Work performed by United Solar Systems Corporation, Troy, Michigan. Available NTIS: Order No. DE93010046.

Herwig, L. O.; Witt, C. E.; Mitchell, R. L.; Mooney, G. D.; Hasti, D.; Sellers, R. (April 1993) "Report on First-Year Results of the Photovoltaic Manufacturing Technology (PVMaT) Phase 2A Process-Specific R\&D Projects." Proceedings of the 1993 Annual Conference of the American Solar Energy Society.

Horner, G. S.; Mascarenhas, A.; Froyen, S.; Alonso, R. G.; Bertness, K.; Olson, J. M. (February 1993). "Photoluminescence-Excitation-Spectroscopy Studies in Spontaneously Ordered GaInP2." Physical Review B (47:7); American Physical Society; pp 4041-4043. TP-451-5170.

Izu, M. (April 1993). Continuous Roll-to-Roll a-Si Photovoltaic Manufacturing Technology, Semiannual Technical Progress Report, 1 April 1992 - 30 September 1992. SERI/TP-411-5453. 55 pp. Work performed by Energy Conversion Devices, Troy, Michigan. Available NTIS: Order No. DE93010006. 
Kalejs, J. P. (September 1993). Mobil Solar Energy Corporation Thin EFG Octagons, Annual Subcontract Report, 1 April 1992 - 30 September 1992. SERI/TP-411-5738. 32 pp. Work performed by Mobil Solar Energy Corporation, Billerica, Massachusetts. Available NTIS: Order No. DE93018222.

Kemp, M.; Branz, H. M. (1993). "Analytic Solution of Trap-Controlled Tracer Diffusion in Amorphous Solids." Physical Review B, Vol. 47, pp. 7067-7070.

Laks, D. B.; Ferreira, L. G.; Froyen, S.; Zunger, A. (November 1992). "Efficient Cluster Expansion for Substitutional Systems." Physical Review B (46:19); American Physical Society; pp. 587-605. TP-451-4956.

Laks, D. B.; Wei, S.-H.; Zunger, A. (December 1992). "Evolution of Alloy Properties with Long-Range Order." Physical Review Letters (69:26); American Physical Society; pp. 3766-3769. NREL/TP-451-5091.

Lu, Z. W.; Zunger, A.; Deutsch, M. (April 1993). "Electronic Charge Distribution in Crystalline Diamond, Silicon, and Germanium." Physical Review B (47:15); pp. 9385-9410. NREL/TP-451-4957.

Lu, Z. W.; Wei, S.-H.; Zunger, A. (January 1993). "Relativity-Induced Ordering and Phase Separation in Intermetallic Compounds." Europhysics Letters (21:2); pp. 221-226.

Lucovsky, G. (August 1993). Fundamental Studies of Defect Generation in Amorphous Silicon Alloys Grown by Remote Plasma-Enhanced Chemical-Vapor Deposition, Final Subcontract Report, 1 July 1989-31 December 1992. SERI/TP-451-5452. 57 pp. Work performed by North Carolina State University, Raleigh, North Carolina. Available NTIS: Order No. DE93017083.

Lucovsky,G.; Nemanich, R. J.; Bernholc, J.; Whitten, J.; Wang, C.; Davidson, B.; Williams, M.; Lee, D.; Bjorkman, C.; Jing, Z. (January 1993). Fundamental Studies of Defect Generation in Amorphous Silicon Alloys Grown by Remote Plasma-Enhanced Chemical Vapor Deposition (Remote PECVD), Annual Subcontract Report, 1 September 1990 - 31 August 1991. SERI/TP-451-4852. $56 \mathrm{pp}$. Work performed by North Carolina State University, Raleigh, North Carolina. Available NTIS: Order No. DE92010560.

Luft, W.; Tsuo, Y. S. (1993). "Hydrogenated Amorphous Silicon Alloy Deposition Processes." Marcel Dekker, Inc., New York, New York.

Luft, W. (1993). "NREL/Industry Interaction: Amorphous Silicon Research Team Formation." American Institute of Physics Conference Proceedings. 
Luft, W.; von Roedern, B.; Stafford, B.; Mrig, L. (1993). "Second Controlled Light-Soaking Experiment for Amorphous Silicon Modules." Conference Record of the 23rd IEEE PV Specialists Conference, pp. 860-866.

Lundstrom, M. S.; Melloch, M. R.; Pierret, R. F.; Carpenter, M. S.; Chuang, H. L.; Dodd, P. E.; Keshavarzi, A.; Klausmeier-Brown, M. E.; Lush, G. B.; Stellwag, T. B. (January 1993). Basic Studies of III-V High Efficiency Cell Components, Annual Subcontract Report, 15 August 1989 - 14 August 1990. SERI/TP-451-4850. 67 pp. Work performed by Purdue University, West Lafayette, Indiana. Available NTIS: Order No. DE92010563.

Lundstrom, M. S.; Melloch, M. R.; Lush, G. B.; O'Bradovich, G. J.; Young, M. P. (January 1993). New III-V Cell Design Approaches for Very High Efficiency, Annual Subcontract Report, 1 August 1990 - 31 July 1991. SERI/TP-451-4851. 53 pp. Work performed by Purdue University, West Lafayette, Indiana. Available NTIS: Order No. DE92010558.

Lundstrom, M. S.; Melloch, M. R.; Lush, G. B.; Patkar, M. P.; Young, M. P. (April 1993). New III-V Cell Design Approaches for Very High Efficiency, Annual Subcontract Report, 1 August 1991 - 31 July 1992. SERI/TP-451-5355. $117 \mathrm{pp}$. Work performed by Purdue University, West Lafayette, Indiana. Available NTIS: Order No. DE93000097.

Mahan, A. H.; Johnson, E. J.; Webb, J. D. (April 1993). "An Examination of H Effusion in aSi:H Using Infrared Spectroscopy." Proceedings of the MRS Symposium on Amorphous Silicon Technology.

Mason, A. V.; Lillington, D. R. (October 1992). Photovoltaic Manufacturing Technology Report, Phase 1, Final Technical Report, 9 January 1991 - 14 April 1991. SERI/TP-411-5019. 135 pp. Work performed by Spectrolab, Inc., Sylmar, California. Available NTIS: Order No. DE93000027.

McMahon, T. J.; Xiao, Y. "Electron Spin Resonance Study of the Dangling Bond in Amorphous Si and Porous Si." Applied Physics Letters 63, p. 1657.

Mitchell, K. W.; Eberspacher, C. (February 1993). Research on High Efficiency, Large-Area CuInSe2-Based Thin-Film Modules, Annual Subcontract Report, 1 May 1991 - 30 April 1992. SERI/TP-413-5332. $56 \mathrm{pp}$. Work performed by Siemens Solar Industries, Camarillo, California. Available NTIS: Order No. DE93000077.

Morel, D. L.; Attar, G.; Karthikeyan, S.; Muthaiah, A.; Zafar, A. (August 1993). Advanced Processing Technology for High-Efficiency, Thin-Film CuInSe2 Solar Cells, Annual Subcontract Report, 1 March 1992 - 28 February 1993. SERI/TP-451-5653. 38 pp. Work performed by University of South Florida, Tampa, Florida. Available NTIS: Order No. DE93010045. 
Myers, D. R.; Cannon, T. W.; Trudell, D. (September 1993). "Radiometric Measurements for PV Characterization." Photovoltaics Performance and Reliability Workshop, September 8-10, 1993, Golden, CO. NREL/TP-411-5957.

Nelson, A. J.; Frigo, S.; Rosenberg, R. A. (1993). "Photoemission Investigation on the Effect of $\mathrm{H}_{2} \mathrm{~S}$ Plasma Exposure of InP." Surface Chemical Cleaning and Passivation for Semiconductor Processing, Materials Research Society Conference Proceedings No. 315.

Nelson, A. J.; Schwerdtfeger, C. R.; Wei, S.-H.; Zunger, A.; Rioux, D.; Patel, R.; Hochst, H. (1993). "Theoretical and Experimental Studies of the $\mathrm{ZnSe} / \mathrm{CuInSe}{ }_{2}$ Heterojunction Band Offset." Applied Physics Letters 62 (20), p. 2557.

Nelson, A. J.; Frigo, S.; Rosenberg, R. A. (1993). "Surface Type Conversion of InP by $\mathrm{H}_{2} \mathrm{~S}$ Plasma Exposure: A Photoemission Investigation." Journal of Vacuum Science Technology A11(4), p. 1022.

Nelson, A. J.; Frigo, S. P.; Rosenberg, R. (December 1992). "Photoemission Characterization of H2 Plasma Etched Surface of CdS." Journal of Applied Physics (72:12); American Institute of Physics; pp. 5881-5887. TP-412-4977.

Nelson, A. J.; Frigo, S.; Rosenberg, R. A. (1993). "Valency and Type Conversion in CuInSe With $\mathrm{H}_{2}$ Plasma Exposure: A Photoemission Investigation." Journal of Applied Physics, 73(12), p. 8561.

Nelson, A. J.; Niles, D. W.; Rioux, D.; Patel, R.; Hochst, H. (December 1992). "Soft X-Ray Photoemission Investigation on the Effect on In Doping in CdS/CuInSe2 Heterojunction Formation." Journal of Applied Physics (72:12); American Institute of Physics; pp. 5888-5891.

Niles, D. W.; Rioux, D.; Hochst, H. (May 1993). "Photoemission Investigation of the SnO2/CdS Interface: A Front Contact Interface Study of CdS/CdT Solar Cells." Journal of Applied Physics (73:9); American Institute of Physics; pp. 4586-4590. NREL/TP-412-5095.

Nolan, J. F.; Meyers, P. V. (August 1993). Fabrication of Stable, Large-Area Thin-Film CdTe Photovoltaic Modules, Annual Subcontract Report, 10 May 1992 - 9 May 1993. SERI/TP-413-5740. 28 pp. Work performed by Solar Cells, Inc., Toledo, Ohio. Available NTIS: Order No. DE93018204.

NREL Photovoltaic Subcontract Reports: Abstracts and Document Control Information, 1 August 1992- 31 July 1993. (September 1993). SERI/TP-410-5812. 65 pp. Available NTIS: Order No. DE93018218. 
Olson, J. M.; Kurtz, S. R., Inventors (June 1993). Current-Matched High-Efficiency, Multijunction Monolithic Solar Cells, U.S. Patent No. 5,223,043. 26 pp. Assignee: U.S. Department of Energy.

Olson, J. M.; Kurtz, S. R., Inventors (November 1992). Tunnel Junction Multiple Wavelength Light-Emitting Diodes, U.S. Patent No. 5,166,761. 4 pp. Assignee: Midwest Research Institute, Kansas City, Missouri.

Osorio, R.; Froyen, S. (January 1993). "Interaction Parameters and a Quenched-Disorder Phase Diagram for (GaAs) 1-x Ge2x Alloys." Physical Review B (47:4); American Physical Society; pp. 1889-1897. TP-451-5004.

Osorio, R.; Lu, Z.-W.; Wei, S.-H.; Zunger, A. (April 1993). "First-Principles Phase Diagrams of Pseudoternary Chalioprite/Zinc-Blende Alloys." Physical Review B, Rapid Communications (47:15); American Physical Society; pp. 9985-9988. NREL/TP-451-5194.

Oswald, R.; O'Dowd, J. (April 1993). Large-Area, Triple-Junction a-Si Alloy Production Scale-Up, Semiannual Technical Progress Report, 17 March 1992 - 18 September 1992. SERI/TP-411-5454. 31 pp. Work performed by Solarex Thin Film Division, Newtown, Pennsylvania. Available NTIS: Order No. DE93010007.

Paul, W. (April 1993). Structural and Electronic Studies of a-SiGe:H Alloys, Final Subcontract Report, 1 January 1991 - 28 February 1993. SERI/TP-411-5457. 64 pp. Work performed by Harvard University, Cambridge, Massachusetts. Available NTIS: Order No. DE93010021.

Peterson, T. M.; Luft, W. (1993). "Adopting a Customer-Focused Team Approach to Amorphous Silicon Multijunction Module R\&D." Conference Record of the 23rd IEEE PV Specialists Conference, pp. 950-954.

Photovoltaic Advanced Research and Development Project, Technical Summary Report, August, 1993. (September 1993). SERI/MP-410-5837. 54 pp.

Photovoltaic Advanced Research and Development Project, Technical Summary Report, May 1993. (June 1993). SERI/MP-410-5649. 55 pp.

Photovoltaic Advanced Research and Developlent Project, Technical Summary Report, July 1993. (August 1993). SERI/MP-410-5758. 55 pp.

Photovoltaic Advanced Research and Development Project, Technical Suymmary Report, September 1992. (October 1992). SERI/MP-410-5164. 45 pp.

Photovoltaic Advance Research and Development Project, Technical Summary Report, November 1992. (December 1992). SERI/TP-410-5329. $59 \mathrm{pp.}$ 
Photovoltaic Advanced Research and Development Project, Technical Summary Report, April 1993. (April 1993). SERI/MP/410-5610. 57 pp.

Photovoltaic Advanced Research and Development Project, Technical Summary Report, February 1993. (March 1993). SERI/TP-410-5447. 54 pp.

Photovoltaic Advanced Research and Development Project, Technical Summary Report, January 1993. (February 1993). SERI/MP-410-5411. 55 pp.

Photovoltaic Advanced Research and Development Project, Technical Summary Report, December 1992. (January 1993). SERI/MP-410-5367. 47 pp.

Photovoltaic Advanced Research and Development Project, Technical Summary Report, March 1993. (April 1993). SERI/MP-410-5562. 55 pp.

Photovoltaic Manufacturing Technology Phase 1, Final Technical Report, 1 May 1991 - 10 May 1991. (October 1992). SERI/TP-411-5018. 49 pp. Work performed by Chronar Corporation, Lawrenceville, New Jersey. Available NTIS: Order No. DE93000026.

Polycrystalline Thin Films FY92 Project Report. (January 1993). SERI/TP-413-5270. 19 pp. Available NTIS: Order No. DE93000070.

Pugh, J. R.; Mao, D.; Zhang, J.-G.; Heben, M. J.; Nelson, A. J.; Frank, A. J. (1993). "A Metal:p-n-CdTe Schottky-Barrier Solar Cell: Photoelectrochemical Generation of a Shallow P-Type Region in n-CdTe." Journal of Applied Physics 74(4), p. 2619.

Qu, Y.; Gessert, T. A.; Ramanathan, K.; Dhere, R. G.; Noufi, R.; Coutts, T. J. (1993). "Electrical and Optical Properties of Ion Beam Sputtered ZnO:Al films as a Function of Film Thickness." Journal of Vacuum Science Technology A 11(4).

Rand, J. A.; Cotter, J. E.; Ingram, A. E.; Ruffins, T. R.; Shreve, K. P.; Hall, R. B.; Barnett, A. M. (June 1993). Development of Large-Area Monolithically Integrated Silicon-Film Photovoltaic Modules, Annual Subcontract Report, 16 November 1991 - 31 December 1992. SERI/TP-413-5581. 23 pp. Work performed by AstroPower, Inc., Newark, Delaware. Available NTIS: Order No. DE93010037.

Rohatgi, A.; Sudharsanan, R.; Ringel, S. A.; Chou, H. C. (October 1992). High Efficiency Cadmium Telluride and Zinc Telluride Based Thin-Film Solar Cells, Annual Subcontract Report, 1 March 1990 - 28 Februawry 1992. SERI/TP-451-4999. 108 pp. Work performed by Georgia Institute of Technology, Atlanta, Georgia. Available NTIS: Order No. DE92016403. 
Rohatgi, A.; Chou, H. C.; Bhat, A. K.; Kamra, S. (August 1993). Development of High-Efficiency CdTe Solar Cells, Annual Subcontract Report, 1 February 1992 - 31 December 1992. SERI/TP-451-5650. $56 \mathrm{pp}$. Work performed by Georgia Institute of Technology, Atlanta, Georgia. Available NTIS: Order No. DE93018206.

Schiff, E. A.; Gu, Q.; Wang, Q. (August 1993). Research on Defects and Transport in Amorphous-Silicon-Based Semiconductors, Annual Subcontract Report, 20 February 1992 19 February 1993. SERI/TP-451-5651. 19 pp. Work performed by Syracuse University, Syracuse, New York. Available NTIS: Order No. DE93017117.

Sharps, P. R. (April 1993). Growth and Development of GaInAsP for Use in High-Efficiency Solar Cells, Annual Subcontract Report, 1 July 1991 - 30 June 1992. SERITP-451-5414. 23 pp. Work performed by Research Triangle Institute, Research Triangle Park, North Carolina. Available NTIS: Order No. DE93000098.

Shing, Y.-H. (October 1992). Electron Cyclotron Resonance Deposition of Amorphous Silicon Alloy Films and Devices, Final Subcontract Report, 1 April 1991 - 31 March 1992. SERI/TP-411-5123. 58 pp. Work performed by Jet Propulsion Laboratory, Pasadena, California. Available NTIS: Order No. DE93000023.

Sites, J. R. (November 1992). Role of Polycrystallinity in CdTe and CuInSe2 Photovoltaic Cells, Annual Subcontract Report, 1 April 1991 - 31 March 1992. SERI/TP-451-5190. 33 pp. Available NTIS: Order No. DE93000045.

Sobol, P. E.; Nelson, A. J.; Schwerdtfeger, C. R.; Stickle, W. F.; Moulder, J. F. (1993). "Single Crystal CuInSe ${ }_{2}$ Analysis by High Resolution XPS." Surface Science Spectra 1(4), p. 393.

Solar 2000 Update - July 1993. (July 1993). SERI/TP-411-5745. 8 pp. Available in limited quantities from Mark Fitzgerald.

Sopori, B. L., Inventor (June 1993). Controlled Metal-Semiconductor Sintering/Alloying by One-Directional Reverse Illumination, U.S. Patent No. 5,223,453. 12 pp. Assignee: U.S. Department of Energy.

Sopori, B. L.; Jones, K.; Deng, X. J. (November 1992). "Observation of Enhanced Hydrogen Diffusion in Solar Cell Silicon." Applied Physics Letters (61:21); American Institute of Physics; TP-451-4903.

Sopori, B. L., Inventor (June 1993). Apparatus for Synthesis of a Solar Spectrum, U.S. Patent No. 5,217,285. 14 pp. Assignee: U.S. Department of Energy.

Stafford, B. L.; Taylor, R.; Martin, R. (1993) "PV:BONUS - Developing a U.S. Market of PV in Buildings." Conference Record of the 23rd IEEE PV Specialists Conference. 
Stafford, B. L.; Tracy, C. E.; Benson, D. K.; Nelson, A. J., Inventors (March 1993). High Rate Chemical Vapor Deposition of Carbon Films Using Fluorinated Gases, U.S. Patent No. 5,198,263. 14 pp. Assignee: U.S. Department of Energy.

Stanbery, B. J.; Chen, W. S.; Devaney, W. E.; Stewart, J. M. (November 1992). Research on Polycrystalline Thin Film CuGaInSe2 Solar Cells, Annual Subcontract Report, 3 May 1991 - 2 May 1992. SERI/TP-413-5012. 23 pp Work performed by Boeing Defense \& Space Group, Seattle, Washington. Available NTIS: Order No. DE92016439.

Stoffel, T.; Myers, D. R. (May 1993). "Radiometers, Data Collection, and Processing." EPRI Workshop "Measuring the Solar Resource: Acquisition and Use of Solar Radiation Data." May 25-26, 1993, Denver, CO. Daystar, Inc., Las Cruces, NM.

Street, R. A.; Jackson, W. B.; Johnson, N.; Nebel, C.; Hack, M.; Santos, P.; Thompson, R.; Tsai, C. C.; Walker, J. (December 1992). Research on the Stability, Electronic Properties, and Structure of a-Si:H and Its Alloys, Annual Subcontract Report, 1 June 1991 - 31 May 1992. SERI/TP-451-5192. 66 pp. Work performed by Xerox Palo Alto Research Center, Palo Alto, California. Available NTIS: Order No. DE93000050.

Summers, C. J.; Rohatgi, A.; Torabi, A.; Harris, H. M. (January 1993). New Concepts for High Efficiency Energy Conversion: The Avalanch Heterostructure and Superlattice Solar Cells, A Subcontract Report, 1 June 1987 - 31 January 1990. SERI/TP-410-4960. 116 pp. Work performed by Georgia Tech Research Institute, Atlanta, Georgia. Available NTIS: Order No. DE92010592.

Sunrayce 93: Just the Facts. (March 1993). SERI/MK-411-5413. 4 pp. Available in limited quantities from Sunrayce 93 Headquarters, NREL.

Sunrayce: The Road to Success. (November 1992). SERI/MK-411-5097. $6 \mathrm{pp}$.

Surek, T. (1993) "The State of the Art of Thin-Film Photovoltaics," in: Advances in Solar Energy, Volume 8 (Ed., M. Prince), New York, American Solar Energy Society, pp. 83-103 (also NREL/TP-410-6049; available from NTIS: Order No. DE94000224).

Surek, T. (1993) "Overview of the Status and Progress in Photovoltaics Research in the United States," in: Proceedings of the International Solar Energy Society Solar World Congress, Budapest, Hungary, August 23-27, 1993 (in press).

Trefny, J. U.; Furtak, T. E.; Wada, N.; Williamson, D. L.; Kim, D. (August 1993). Poloycrystalline Thin Film Cadmium Telluride Solar Cells Fabricated by Electrodeposition, Annual Subcontract Report, 20 March 1992 - 19 March 1993. SERI/TP-451-5652. 41 pp. Work performed by Colorado School of Mines, Golden, Colorado. Available NTIS: Order No. DE93018201. 
Tsuo, Y. S.; Stone, J. L., Inventors (March 1993). Erasable, Multiple Level Logic Optical Memory Disk, U.S. Patent No. 5,194,349. 21 pp. Assignee: Midwest Research Institute, Kansas City, Missouri.

Tuttle, J.; Contreras, M.; Tennant, A.; Albin, D.; Noufi, R. (1993). "High Efficiency Thin-Film $\mathrm{Cu}(\mathrm{In}, \mathrm{Ga}) \mathrm{Se}_{2}$-Based Photovoltaic Devices: Progress Towards a Universal Approach to Absorber Fabrication." Conference Record of the 23rd IEEE PV Specialists Conference, May 10-14, 1993, Louisville, KY.

Ullal, H. S.; Zweibel, K.; von Roedern, B. G. (August 1993). Thin-Film CdTe and CuInSe2 Photovoltaic Technologies. SERI/TP-413-5752. 6 pp. Available NTIS: Order No. DE93018217.

Vanecek, M.; Mahan, A. H.; Nelson, B. P.; Crandall, R. S. (January 1993). "Influence of Hydrogen and Microstructure on Increased Stability of Amorphous Silicon." Proceedings of the 11th E.C. Photovoltaic Solar Energy Conference, 12-16 October 1992, Montreux, Switzerland, Gunmaressm L.; Palz, W.;DeRezy, C.;Kiess, H.;Helin, P., eds. Harwood Academic Publishers, Switzerland; pp. 96-99.

Venkatasubramanian, R. (January 1993). Inverted AlGaAs/GaAs Patterned-Ge Tunnel Junction Cascade Concentrator Solar Cell, Final Subcontract Report, 1 January 1991 - 31 August 1992. SERI/TP-411-5289. 74 pp. Work performed by Research Triangle Institute, Research Triangle Park, North Carolina. Available NTIS: Order No. DE93000073.

Vernon, S. M. (April 1993). Low-Cost, High-Efficiency Solar Cells Utilizing GaAs-on-Si Technology, Annual Subcontract Report, 1 August 1991 - 31 July 1992. SERI/TP-451-5353. $31 \mathrm{pp}$. Work performed by Spire Corporation, Bedford, Massachusetts. Available NTIS: Order No. DE93000096.

Weaver, P. F.; Frank, A. J., Inventors (May 1993). Reversibly Immobilized Biological Materials in Monolayer Films on Electrodes, U.S. Patent No. 5,208,154. 9 pp. Assignee: U.S. Department of Energy.

Wei, S.-H. (April 1993). "Electronic Structure of II-VI Semiconductor and Their Alloys in II-VI Semiconductor Compounds." M. Jain, Editor. World Scientific, Singapore, p. 71.

Wei, S.-H.; Zhang, S.-B.; Zunger, A. (March 1993). "Off-Center Atomic Displacements in ZincBlende Semiconductors." Physical Review Letters 70, p. 1639.

Wei, S.-H.; Zunger, A. (September 1993). "Electronic Origin of Magnetic Phase Transition in Zinc-Blende Mn Chalcogenides." Physical Review B 48, p. 6111. 
Wei, S.-H.; Laks, D. B.; Zunger, A. (April 1993). "Dependence of the Optical Properties of Semiconductor Alloys on the Degree of Long-Range Order." Applied Physics Letters, 62, p. 1937.

Wells, C. V. (November 1992). Principles and Applications of Measurement Uncertainty Analysis in Research and Calibration. SERI/TP-411-5165. $19 \mathrm{pp}$. Prepared for the 3rd Annual Infrared Radiometric Sensor Calibration Symposium, 14-17 September 1992, Logan, Utah. Available NTIS: Order No. DE93000034.

Wells, C. (October 1992). Measurement Uncertainty Analysis Techniques Applied to PV Performance Measurements. SERI/TP-411-5125. $18 \mathrm{pp}$. Prepared for the Photovoltaics Performance and Reliability Workshop, 16-18 September 1992, Golden, Colorado, also published as pp 379-396 of the conference proceedings. Available NTIS: Order No. DE93000019.

Whisnant, R. A.; Sherring, C. (August 1993). Amorphous Silicon Batch Process Cost Analysis, Annual Subcontract Report, 11 March 1991 - 28 February 1993. SERI/TP-411-5644. 94 pp. Work performed by Research Triangle Institute, Research Triangle Park, North Carolina. Available NTIS: Order No. DE93018203.

Witt, C. E.; Mooney, G. D.; Mitchell, R. L. (August 1993) "The Photovoltaic Manufacturing Technology (PVMaT) Project." Proceedings of the 29th National Heat Transfer Conference, Atlanta, GA.

Witt, C. E.; Mitchell, R. L.; Mooney, G. D.; Herwig, L. O.; Hasti, D.; Sellers, R. (May 1993) "Progress in Phases 2 and 3 of the Photovoltaic Manufacturing Technology Project (PVMaT)." Proceedings of the 23rd IEEE PVSC, Louisville, KY.

Yeh, C.-Y.; Lu, Z. W.; Froyen, S.; Zunger, A. (November 1992). "Zinc-blende - Wurtzite Polytypism in Semiconductors." Physical Review B (46:16); The American Physical Society; pp 10086 - 10097 . NREL/TP-451-4839.

Yeh, C.-Y.; Lu, Z. W.; Froyen, S.; Zunger, A. (October 1992). "Zinc-Blende-Wurtzite Polytypism in Semiconductors." Physical Review B, Volume 46, Number 16.

Yelon, A.; Movaghar, B.; Branz, H. M. (1992). "Origin and Consequences of the Compensation (Meyer-Neldel) Law." Physical Review B, Vol. 46, pp. 12244-12250.

Zhang, S. B.; Zunger, A. (September 1993). "Predictions of Unusual Electronic Properties of Si Quantum Films." Applied Physics Letters 63, p. 1399.

Zhang, S. B.; Zunger, A. (September 1993). "Prediction of Unusual Electronic Properties of Si Quantum Films." Applied Physics Letters, 63(10), p. 1399. 
Zunger, A.; Wagner, S.; Petroff, P. M. (January 1993). "New Materials and Structures for Photovoltaics." Journal of Electronic Materials (22:1); pp 3-16. TP-451-5020.

Zweibel, K.; Chu, T. L.; Chu, S. S. (1993). "CdTe PV." Chapter 16 in Advances in Solar Energy, (M. Prince, Editor). Volume 8, ASES.

Zweibel, K.; Ullal, H. S.; von Roedern, B. G.; Noufi, R.; Coutts, T. J.; Al-Jassim, M. M. (1993). "Perspectives and Opportunities in Polycrystalline Thin-Film Photovoltaic Technologies." Conference Record of the 23rd IEEE PV Specialists Conference, p. 379-388. 


\begin{tabular}{|c|c|c|c|}
\hline $\begin{array}{l}\text { Document Control } \\
\text { Page }\end{array}$ & $\begin{array}{l}\text { 1. NREL Report No. } \\
\text { NREL/TP-411-6270 }\end{array}$ & $\begin{array}{l}\text { 2. NTIS Accession No. } \\
\text { DE } 94000272\end{array}$ & 3. Recipient's Accession No. \\
\hline \multirow{3}{*}{\multicolumn{3}{|c|}{$\begin{array}{l}\text { 4. Title and Subtitle } \\
\text { NREL Photovoltaic Program FY } 1993 \text { Bibliography }\end{array}$}} & 5. Publication Date \\
\hline & & & January 1994 \\
\hline & & & 6. \\
\hline \multicolumn{3}{|l|}{$\begin{array}{l}\text { 7. Author(s) } \\
\text { L. Pohle, Editor }\end{array}$} & 8. Performing Organization Rept. No. \\
\hline \multicolumn{3}{|c|}{$\begin{array}{l}\text { 9. Performing Organization Name and Address } \\
\text { National Renewable Energy Laboratory }\end{array}$} & $\begin{array}{l}\text { 10. Project/TaskWork Unit No. } \\
\text { PV410101 }\end{array}$ \\
\hline \multicolumn{3}{|c|}{ National Renewable Energy Laboratory } & $\begin{array}{l}\text { 11. Contract (C) or Grant (G) No. } \\
\text { (C) } \\
\text { (G) }\end{array}$ \\
\hline \multirow{2}{*}{\multicolumn{3}{|c|}{$\begin{array}{l}\text { 12. Sponsoring Organization Name and Address } \\
\text { National Renewable Energy Laboratory } \\
1617 \text { Cole Blvd. } \\
\text { Golden, CO } 80401-3393\end{array}$}} & $\begin{array}{l}\text { 13. Type of Report \& Period Covered } \\
\text { Technical Report } \\
\text { October } 1992 \text { - September } 1993 \\
\end{array}$ \\
\hline & & & 14. \\
\hline \multicolumn{4}{|c|}{$\begin{array}{l}\text { 15. Supplementary Notes } \\
\text { NREL technical monitor: NA }\end{array}$} \\
\hline \multicolumn{4}{|c|}{$\begin{array}{l}\text { This report lists all published documents of the Photovoltaic Program for FY 1993. Documents include conference papers, } \\
\text { journal articles, book chapters, etc. }\end{array}$} \\
\hline \multicolumn{4}{|c|}{$\begin{array}{l}\text { 17. Document Analysis } \\
\text { a. Descriptors } \\
\text { photovoltaics ; solar cells } \\
\text { b. Identifiers/Open-Ended Terms } \\
\text { c. UC Categories } \\
270\end{array}$} \\
\hline \multirow{2}{*}{\multicolumn{3}{|c|}{$\begin{array}{l}\text { 18. Availability Statement } \\
\text { National Technical Information Service } \\
\text { U.S. Department of Commerce } \\
\text { 5285 Port Royal Road } \\
\text { Springfield, VA } 22161\end{array}$}} & $\begin{array}{l}\text { 19. No. of Pages } \\
18 \\
\end{array}$ \\
\hline & & & $\begin{array}{l}\text { 20. Price } \\
\text { A03 }\end{array}$ \\
\hline
\end{tabular}

Form No. 0069E (6-30-87) 